\title{
Retrospective Seroepidemiological Survey for Human Babesiosis in an Area in Japan Where a Tick-Borne Disease is Endemic
}

\author{
Satoru ARAI ${ }^{1)}$, Masayoshi TSUJI ${ }^{3)}$, ${\text { Ikuo } \mathrm{KAIHO}^{4)} \text {, Haruka MURAYAMA }}^{3)}$, Aya ZAMOTO $^{3)}$, Qiang $\mathrm{WEI}^{3)}$, \\ Nobuhiko OKABE ${ }^{1)}$, Tsuneo KAMIYAMA ${ }^{2)}$ and Chiaki ISHIHARA ${ }^{3)}$ \\ ${ }^{1)}$ Infectious Disease Surveillance Center and ${ }^{2)}$ Department of Veterinary Science, National Institute of Infectious Diseases, Shinjuku, \\ Tokyo 162-8640, ${ }^{3)}$ School of Veterinary Medicine, Rakuno Gakuen University, Ebetsu 069-8501 and ${ }^{4)}$ Public Health Laboratory of Chiba \\ Prefecture, 666-2 Nitona, Chuo-ku, Chiba 260-8715, Japan
}

(Received 5 August 2002/Accepted 10 December 2002)

ABSTRACT. A total of 1,335 archived human sera collected in 1985 from an area in Japan where a tick-borne disease is endemic were examined by indirect immunofluorescence antibody test (IFAT) to estimate seroprevalence against three serologically distinct types of Babesia microti-like parasites; namely, Hobetsu, Kobe, and U.S. types. Eighteen sera (1.3\%) were found to be IFAT-positive (titer 1:100-1:6,400), of which 14 and three were ascertained by Western blot analysis to be positive against the Hobetsu and Kobe types, respectively. In addition, four sera showed an IFAT titer of 1:100 against the U.S. type, but they appeared to be false-positive because they were cross-reactive against the Hobetsu and Kobe types, and also because a U.S.-type parasite has not been found in Japan. Our results suggest that human babesiosis in Japan occurred prior to the discovery of the index case in 1999 and that the infections were caused mainly by Hobetsu-type parasites.

KEY WORDS: Babesia microti, human babesiosis, Japan, serosurvey, tick-borne disease.

J. Vet. Med. Sci. 65(3): 335-340, 2003

Human babesiosis is a tick-transmitted zoonosis caused by intraerythrocytic protozoan parasites of the genus Babesia $[5,8,21]$. The cases of human babesiosis reported to date include over 20 caused by Babesia divergens in Europe [5] and hundreds with additional tens per year caused by $B$. microti in the United States [8]. Meanwhile, no human cases were reported in Japan until quite recently, despite the clearly documented presence of $B$. microti-like parasites among the large Japanese field mice, Apodemus speciosus [20].

In our previous studies $[13,19]$, we investigated the first case of transfusion-acquired, symptomatic human babesiosis in Japan, which occurred in Kobe, Hyogo Prefecture, in 1999. Seemingly identical B. microti-like parasites (designated as the Kobe type) were isolated from both the patient and an asymptomatic carrier who had donated the units of blood that were transfused into the patient. The same type of parasite has also been found in Apodemus mice captured near the donor's residential area [22, 23]. More recently, however, we have isolated a number of $B$. microti-like parasites from wild rodents captured at various places in Japan, and found that another type of parasite (designated as the Hobetsu type), which differs antigenically and genotypically from the Kobe type, is the major type distributed among Japanese small wild rodents [22]. Thus, at least two types of indigenous B. microti-like parasites (Kobe and Hobetsu types) exist in Japan, both of which are phylogenetically related closely to, but antigenically distinct from, $B$. microti sensu stricto (designated as the U.S. type in this

\footnotetext{
* Correspondence to: Tsuji, M., School of Veterinary Medicine, Rakuno-Gakuen University, 582-1 Bunkyodai-midorimachi, Ebetsu 069-8501, Hokkaido, Japan.
}

study) which is distributed in regions of northeastern United States where human babesiosis is endemic.

Although epidemiological information on human babesiosis currently available in Japan is still very limited, evidence reported by Shiota et al. [20] nearly two decades ago, together with that currently accumulating in our ongoing studies, suggests that the infection cycles between the rodent reservoir and the tick vector of these two types of $B$. microti-like parasites may have been established in Japan long before. Even though no human babesiosis case had been reported in Japan before 1999, the infections in humans might have simply been undetected for many years. In the present study, therefore, we conducted a retrospective seroepidemiological survey to obtain further evidence to support this point of view, and also to obtain an estimate of seroprevalence among Japanese. Another aim of this study was to determine whether there is any human serum that contains specific antibodies against the Hobetsu-type $B$. microti, because susceptibility of humans to this newly identified parasite has not been determined yet.

\section{MATERIALS AND METHODS}

Serum samples: The sera examined in the present study were obtained from 1,335 residents (average age of 57.4, ranging from 21 to 89 ; 476 males, 776 females and 83 unidentified) in a rural area of southern Boso Peninsula, Chiba Prefecture, Japan. The serum samples were collected in 1985 for serosurvey of Japanese Spotted Fever [12], a ticktransmitted rickettsiosis that is endemic in that area [6], and have been stored since then at $-20^{\circ} \mathrm{C}$.

Parasites: Two Japanese $B$. microti-like parasites, namely, strains Ko524 and Ho234, and a B. microti isolate 
from the United States, the Gray strain, were used in this study. Strain Ko524 [19] was isolated from the Japanese index case patient in Kobe City, Hyogo Prefecture, Japan [13]. Strain Ho234 was isolated from a large Japanese field mouse (Apodemus speciosus) captured in Hokkaido [23]. These two Japanese strains were serologically distinct, and were used as the type strains of Kobe- and Hobetsu-type parasites, respectively. The Gray strain was originally isolated from the United States index case patient [4], and was used as the type strain of U.S.-type B. microti. All the parasites were propagated in splenectomized hamsters, from which parasitized erythrocytes were obtained when they had 30-50\% parasitemia.

Indirect immunofluorescent antibody test (IFAT): The method of IFAT has previously been described [19]. Two steps of screening tests were performed for selection of antibody-positive sera. For the primary screening, antigencoated IFAT slides were prepared with a mixture of erythrocytes infected with each of strains Ko524, Ho234 and Gray. The mixed erythrocytes had a parasitemia of $45 \%$ (approximately $15 \%$ for each one of the three strains). Thin smeared blood film was made by spreading the erythrocytes in each well of a 24-well HT Coating Slide (MS 342 BL; Bokusui Brown, Tokyo, Japan). For the secondary screening and subsequent antibody titration, IFAT slides were prepared individually with each one of the three strains. In both the primary and secondary screening tests, serum samples were diluted 1:25 with phosphate bufferd saline (PBS), $\mathrm{pH}$ 7.2, containing $1 \% \mathrm{BSA}$ and $0.05 \% \mathrm{NaN}_{3}, 15 \mu \mathrm{l}$ of which was added to each well of antigen-coated slides. After 1-hr incubation at room temperature, the slides were washed in PBS, and $15 \mu l$ of fluorescein isothiocyanate-labeled secondary antibody was added to each well. Affinity purified IgG $\mathrm{F}(\mathrm{ab})_{2}$ fragment of goat anti-human IgG, IgA and IgM (Jackson ImmunoResearch Laboratories, Inc., West Grove, Pa.) at 1:100 dilution in 0.1\% BSA-PBS was used as the secondary antibody. The IFAT slides were incubated at room temperature for $1 \mathrm{hr}$, followed by washing in PBS. Component A of the Slowfade antifade kit (Molecular Probes, Eugene, Oreg.) was mounted onto each well, and cover glasses were placed on the slides. Fluorescent parasites in erythrocytes were observed under a fluorescence microscope at a magnification of $200 \times$. Serial 2-fold dilutions starting from 1:25 were made for the sera that were selected through the secondary screening tests, and their antibody titers were determined by IFAT in the same manner.

Western blot analysis: The procedure of Western blot analysis has been described previously [1, 23]. Babesiainfected erythrocytes were lysed at $4^{\circ} \mathrm{C}$ in a solution consisting of $10 \mathrm{mM}$ Tris- $\mathrm{HCl}$ and $10 \mathrm{mM}$ EDTA (pH 7.5), followed by centrifugation at $10,000 \times \mathrm{g}$ for $10 \mathrm{~min}$. The resulting pellets were washed four times in the same solution, and then resuspended in $125 \mathrm{mM}$ Tris- $\mathrm{HCl}(\mathrm{pH}$ 6.5) containing 5\% $\beta$-mercaptoethanol, $2 \%$ sodium dodecyl sulfate, $10 \%$ glycerol, and $0.1 \%$ bromophenolblue. The samples were heated at $98^{\circ} \mathrm{C}$ for $5 \mathrm{~min}$, vigorously vortexed, and subjected to sodium dodecyl sulfate-polyacrylamide gel electrophoresis in $7.5 \%$ acrylamide gels. Proteins were electrophoretically transferred to Fluorotrans membranes (Pall BioSupport, Port Washington, N.Y.). After blocking with PBS containing $0.5 \%$ casein, the membranes were allowed to react overnight with human sera diluted at 1:100, followed by reaction with alkaline phosphatase-conjugated secondary antibodies (affinity purified $\operatorname{IgG~F}\left(\mathrm{ab}^{\prime}\right)_{2}$ fragment of goat anti-human IgG, IgA and IgM; Jackson ImmunoResearch Laboratories, Inc., West Grove, Pa.). Convalescent phase sera from infected hamsters were used as positive control antibodies, which were detected with alkaline phosphatase-conjugated anti-hamster IgG (Jackson ImmunoResearch Laboratories, Inc.). Antibody reaction was visualized by staining with 5 bromo-4-chloro-3indolylphosphate-nitroblue tetrazolium alkaline phosphatase substrates kit IV (Vector Laboratories, Inc., Burlingame, Calif.).

\section{RESULTS}

Survey by IFAT: Two steps of screening IFAT were carried out with a total of 1,335 archived sera collected in 1985 from apparently healthy residents in the southern Boso Peninsula, Chiba Prefecture, Japan. For the primary screening, the sera at 1:25 dilution were tested against a mixed IFAT antigen consisting of strains Ho234 (Hobetsu type), Ko524 (Kobe type) and Gray (U.S. type). This screening gave rise to 60 tentatively positive sera, which were then tested separately against each of the three types. By this secondary screening test, 43 of the 60 sera were found to be reactive at 1:25 dilution against either one or more of the three types. Serial 2-fold dilutions were made with these 43 sera, and antibody titers were determined against the three parasite antigens. Of the 43 sera, 21 showed fluorescent reaction at 1:25 or 1:50, which were taken as the borderline. The other 22 samples exhibited fluorescent reaction at 1:100 or higher, which was considered to be IFAT-positive. Their antibody titers against each of the three parasite antigens are listed in Table 1, which shows that 15 and three sera were positive against the Hobetsu and Kobe types, respectively, and that these sera reacted mainly against a single type of parasite antigen. There were four sera that were fluorescent-positive at 1:100 dilution against the U.S. type, but they were crossreactive against the other types. These sera were considered to be false-positive because of the reasons described in Discussion.

Western blot analysis: The presence of specific antibodies in the 22 IFAT-positive sera was further ascertained by Western blot analysis. Of the 16 sera that exhibited IFAT titers of 1:100 or higher against the Hobetsu type, 14 reacted with an immunodominant $37 \mathrm{kDa}$ antigen of strain Ho234 (Fig. 1). Of the 14 sera, two (nos. 432 and 979) additionally reacted with a $35 \mathrm{kDa}$ antigen. The other two (nos. 371 and 504) that had an IFAT titer of 1:100 against the Hobetsu type showed either no band or bands not corresponding to those seen with a control serum. The antibody titers of IFAT were correlated roughly with the intensities of the 37 
Table 1. Reciprocal antibody titers against three Babesia strains in IFAT

\begin{tabular}{cccccc}
\hline $\begin{array}{c}\text { Sample } \\
\text { no. }\end{array}$ & Sex & Age & $\begin{array}{c}\text { Ho234 } \\
\text { (Hobetsu type) }\end{array}$ & $\begin{array}{c}\text { Ko524 } \\
\text { Kobe type) }\end{array}$ & $\begin{array}{c}\text { Gray } \\
\text { (U.S. type) }\end{array}$ \\
\hline 482 & F & 56 & 6,400 & $<25$ & $<25$ \\
1107 & F & 50 & 1,600 & $<25$ & $<25$ \\
930 & M & 81 & 800 & $<25$ & $<25$ \\
954 & M & 57 & 800 & $<25$ & $<25$ \\
15 & M & 61 & 400 & $<25$ & $<25$ \\
187 & F & 45 & 400 & $<25$ & $<25$ \\
481 & F & 63 & 400 & $<25$ & $<25$ \\
770 & F & 67 & 400 & $<25$ & $<25$ \\
979 & F & 71 & 400 & $<25$ & 50 \\
33 & M & 44 & 200 & 25 & 25 \\
1142 & F & 43 & 200 & 50 & $<25$ \\
128 & F & 64 & 100 & $<25$ & $<25$ \\
432 & F & 52 & 100 & $<25$ & $<25$ \\
504 & F & 50 & 100 & $<25$ & $<25$ \\
1053 & F & 70 & 100 & $<25$ & $<25$ \\
1091 & F & 89 & $<25$ & 800 & $<25$ \\
1097 & F & 74 & $<25$ & 400 & $<25$ \\
1250 & F & Unknown & 50 & 100 & $<25$ \\
371 & M & 61 & 100 & 100 & 100 \\
468 & Unknown & Unknown & 50 & $<25$ & 100 \\
988 & M & 46 & 25 & 25 & 100 \\
1038 & M & 68 & 50 & 25 & 100 \\
\hline & & & & & \\
\hline
\end{tabular}

kDa bands. Three sera (nos. 1091, 1097 and 1250) that had IFAT titers of 1:100 or higher against the Kobe type reacted with an immunodominant $43 \mathrm{kDa}$ protein of Ko524 strain (Fig. 2a). Four sera (nos. 371, 468, 988 and 1038) that had an IFAT titer of 1:100 against the Gray strain did not show any specific band in Western blot analysis with the same parasite antigen (Fig. 2b).

\section{DISCUSSION}

In this retrospective seroepidemiological survey, we were able to obtain evidence suggesting that some Japanese had been exposed to the Hobetsu- or Kobe-type B. microti-like parasites nearly two decades ago. The presence of specific antibodies in humans against a Hobetsu-type parasite is a significant finding, because it suggests that this type of parasite is capable of infecting humans: evidence for this was not previously available because all the Hobetsu-type parasites isolated to date were obtained only from wild rodents, not from humans. The archived sera tested in the present study were collected in 1985, 14 years before the discovery of the first human babesiosis case in Japan [13]. Thus, it became apparent that despite the absence of reported case before 1999 , the disease had long been in the country without being detected. All the sera were obtained from apparently healthy people, but it is not known whether or not those who were found to be sero-positive in the present study had any clinical symptom of babesiosis. Also not known is whether or not these sero-positive individuals were carriers of Babesia parasites. An attempt to detect babesial DNA in the antibody-positive sera by nested PCR was unsuccessful (S. Arai, unpublished data).

The Japanese index case patient who developed severe clinical symptoms of babesiosis [13] proved to have been infected by blood transfusion from an asymptomatic carrier $[19,23]$ who was a resident of Awaji Island of Hyogo Prefecture [22]. Meanwhile, the human sera examined in the present study were obtained from a rural area of southern Boso Peninsula in Chiba Prefecture, which was far away from Awaji Island (approximately $500 \mathrm{~km}$ apart). Even though there is no geographically meaningful connection between these two places, both are known to be the regions

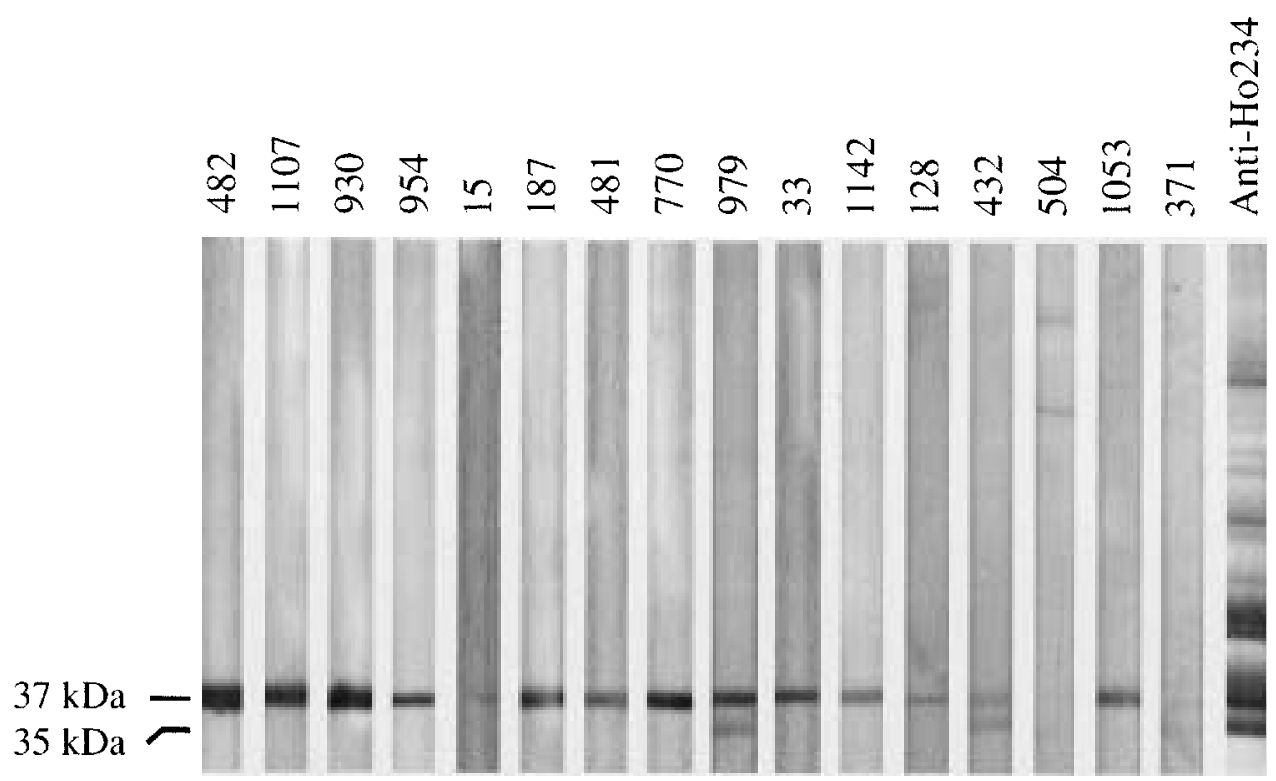

Fig. 1. Western blot analysis of IFAT-positive human sera with strain Ho234. 

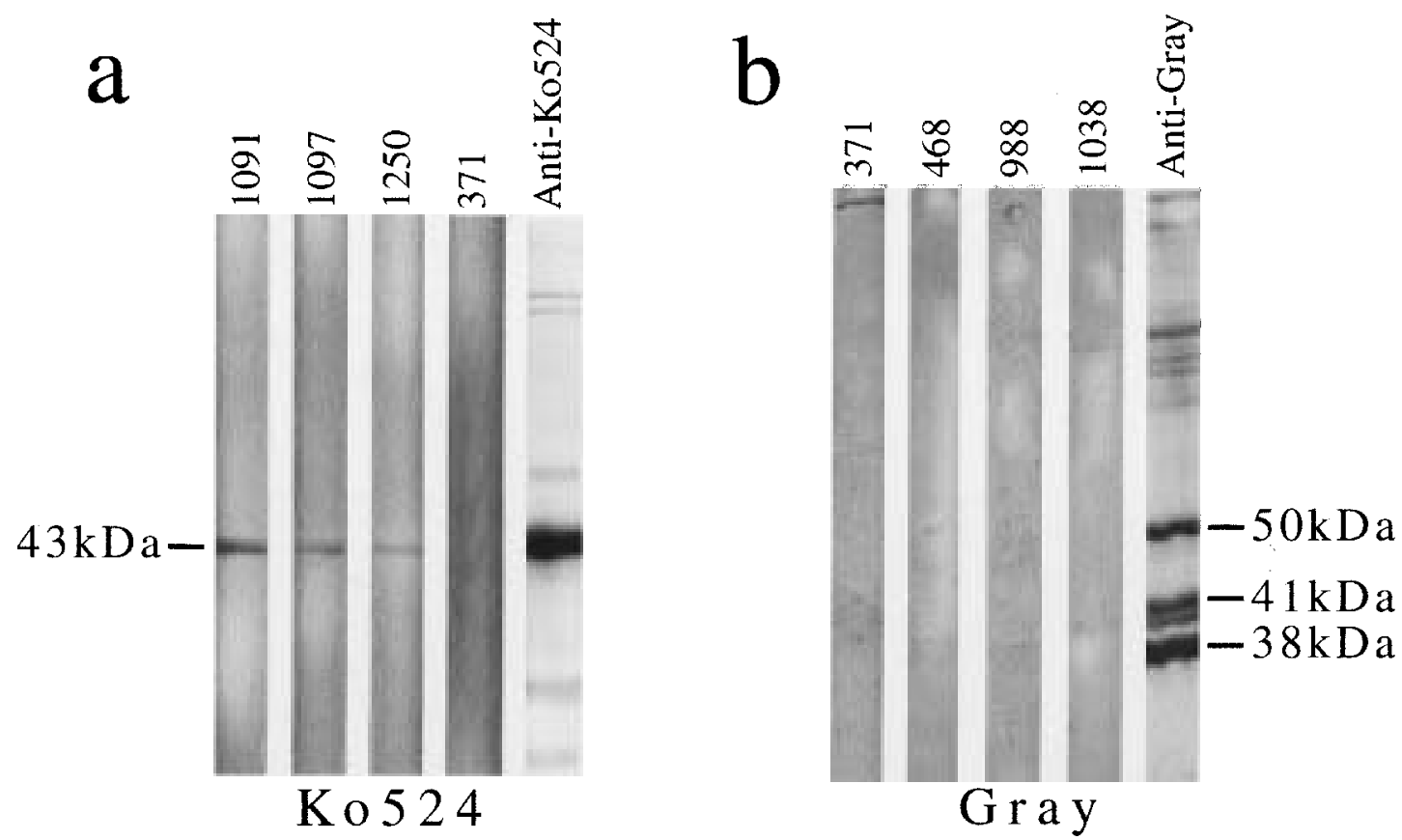

Fig. 2. Western blot analyses of IFAT-positive human sera with strains Ko524 (a) and Gray (b).

where the Japanese Spotted Fever [12], another tick-borne disease caused by Rickettsia japonica, is endemic [6, 7]. In both southern Boso Peninsula and Awaji Island, ticks are abundant during spring and summer in mountainous bushy areas, but whether or not the co-existence of babesiosis and rickettsiosis is linked to the presence of any tick species common in both the places is not clear, as the vector ticks transmitting these two diseases have not been identified yet. In our epizootiologic field surveys [22], we were able to capture wild rodents harboring $B$. microti-like parasites in various places in Japan, including Hokkaido, Chiba, Shiga, Hyogo, Shimane and Tokushima Prefectures. Interestingly, clinical cases of tick-borne diseases have been reported from most of these places; Japanese Spotted Fever from Chiba, Hyogo, Shimane and Tokushima Prefectures [12], and Lyme borreliosis from Hokkaido [15].

In our IFAT, serum samples exhibiting antibody titers of 1:100 or higher were considered to be positive, because those sera exhibited specific fluorescent reaction patterns that are consistent with the shapes of parasite bodies within the erythrocytes. This cut-off level is more conservative than those reported in other studies $[3,9,10]$ or that in the standardized IFAT protocol used in the Centers for Disease Control and Prevention, which is 1:16 or higher. In the present study, as many as 21 additional samples were florescence-positive at 1:25 or 1:50 dilution, but we left these samples as "borderline", because many of them not only exhibited weak fluorescence but also showed a tendency to cross-react against two or three antigenically distinct parasite antigens. This is in contrast to the samples having high IFAT titers (1:200 or higher), which displayed evident fluo- rescence against a single serotype with minimal or no crossreaction against the other types (Table 1). An analogous finding was also obtained when we tested the serum samples against U.S.-type B. microti. We found four sera that had an IFAT titer of 1:100 against a U.S.-type antigen, despite that a parasite belonging to this type has not yet been isolated in Japan [22]. All the four sera showed cross-reaction against either one or both of the Hobetsu and Kobe types, suggesting that our IFAT may not have detected antibodies which were raised by an infection with a U.S.-type parasite, but may have detected antibodies recognizing some widely cross-reactive antigens, such as heat shock proteins [15] and nuclear antigens [16], against which antibodies might be raised by other infectious agents $[15,17]$ or due to some autoimmune diseases [2, 25].

We found that 17 of the 1,335 serum samples $(1.3 \%)$ were unequivocally positive against Japanese $B$. microtilike parasites, based on the results of both IFAT and Western blot analysis. Even though this prevalence rate is lower than those reported from babesiosis-endemic regions in the northeastern United States [3, 9, 11, 18], it was unexpectedly high for us because human babesiosis had long been believed not to exist in Japan. However, since the present study was performed with sera collected from an area where a tick-borne disease is endemic, prevalence rates in other regions of the country may be much lower than that, especially in urban areas where the majority of the Japanese population reside and where tick-borne diseases are not endemic.

The number of sera that were positive against the Hobetsu type was larger than that against the Kobe type; 14 
and three, respectively. This finding was consistent with the results of our previous study [22], which revealed that the Hobetsu type is the predominant type of parasites distributed throughout the country. Thus, it would be expected that Japanese may be exposed to the Hobetsu-type parasites more frequently than to Kobe type. Nonetheless, the first case patient in Japan was infected with Kobe-type, not Hobetsu-type, parasites. Although the exact reason for this occurrence is unknown, it may be that the Hobetsu-type parasites are less virulent than the Kobe type, so many Japanese might have been infected with this type only asymptomatically.

Previously, we speculated that Kobe-type parasites may have emerged very recently because of its confined geographic distribution and the sudden appearance of a human case due to this type [22], but it is probably not the case because the present survey demonstrated the presence of archived sera that were positive against Kobe-type parasites. In both this and previous studies [22], we were unable to obtain any evidence for the presence of U.S.-type parasites in Japan. It may be interesting to find whether or not this apparent absence of U.S.-type B. microti is relevant to the seemingly rare occurrence of symptomatic human babesiosis cases in the country, which is in contrast to the relatively frequent clinical cases reported from endemic areas in the Unites States [24].

ACKNOWLEDGMENTS. We wish to thank T. Hayakawa, the Infectious Disease Surveillance Center, and M. Koura, the Department of Veterinary Science, the National Institute of Infectious Diseases, for their excellent technical assistance. This work was supported in part by Health Science Grants from the Ministry of Health, Labor and Welfare of Japan and Grants-in-Aid from the Ministry of Education, Science and Culture of Japan (projects 12450139 and 13770128), and by Gakujutsu-Frontier Cooperative Research in Rakuno-Gakuen University.

\section{REFERENCES}

1. Arai, S., Tsuji, M., Kim, S.-J., Nakada, K., Kirisawa, R., Ohta, M. and Ishihara, C. 1998. Antigenic and genetic diversities of Babesia ovata in persistently Infected cattle. J. Vet. Med. Sci. 60: $1321-1327$.

2. Cabral, A. R. and Alarcon-Segovia, D. 1997. Autoantibodies in systemic lupus erythematosus. Curr. Opin. Rheumatol. 9: 387392

3. Filstein, M. R., Benach, J. L., White, D. J., Brody, B. A., Goldman, W. D., Bakal, C. W. and Schwarz, R. S. 1980. Serosurvey for human babesiosis in New York. J. Infect. Dis. 141: 518521.

4. Gleason, N. N., Healy, G. R., Western, K. A., Benson, G. D. and Schultz, M. G. 1970. The "Gray" strain of Babesia microti from a human case established in laboratory animals. J. Parasitol. 56: 1256-1257.

5. Gorenflot, A., Moubri, K., Precigout, E., Carcy, B. and Schetters, T. P. 1998. Human babesiosis. Ann. Trop. Med. Parasitol. 92: 489-501.

6. Kaiho, I., Tokieda, M., Tanaka, H. and Kawamura, A. 1992.
Spotted fever group rickettsiosis in Chiba Prefecture. Jpn. J. Assoc. Infect. Dis. 66: 201-205 (in Japanese with an English summary).

7. Kodama, K., Noguchi, T. and Chikahara, Y. 2000. Outbreak of Japanese spotted fever in the southeastern part of Awaji Island. Jpn. J. Assoc. Infect. Dis. 74: 278-279.

8. Krause, P. J. and Telford, S. R., III. 1999. Babesiosis. pp. 236248. In: Protozoal diseases (Gilles, H. M. ed.), Arnold, London, England.

9. Krause, P. J., Telford, S. R., III, Pollack, R. J., Ryan, R., Brassard, P., Zemel, L. and Spielman, A. 1992. Babesiosis: an under diagnosed disease of children. Pediatrics 89: 10451048 .

10. Krause, P. J., Telford, S. R., III, Ryan, R., Conrad, P. A., Wilson, M., Thomford, J. W. and Spielman, A. 1994. Diagnosis of babesiosis: evaluation of a serologic test for the detection of Babesia microti antibody. J. Infect. Dis. 169: 923-926.

11. Krause, P. J., Telford, S. R., III, Ryan, R., Hurta, A. B., Kwasnik, I., Luger, S., Niederman, J., Gerber, M. and Spielman, A. 1991. Geographical and temporal distribution of babesial infection in Connecticut. J. Clin. Microbiol. 29: 1-4.

12. Mahara, F. 1997. Japanese spotted fever: report of 31 cases and review of the literature. Emerg. Infect. Dis. 3: 105-111.

13. Matsui, T., Inoue, R., Kajimoto, K., Tamekane, A., Katayama, Y., Shimoyama, M., Chihara, K., Saito-Ito, A. and Tsuji, M. 2000. First documentation of human babesiosis in Japan. Jpn. J. Clin. Hematol. 41: 628-634 (in Japanese with an English summary).

14. Mollenhauer, J. and Schulmeister, A. 1992. The humoral immune response to heat shock proteins. Experientia 15: 644 649.

15. Nakao, M., Miyamoto, K., Fukunaga, M., Hashimoto, Y. and Takahashi, H. 1994. Comparative studies on Borrelia afzelii isolated from a patient of Lyme disease, Ixodes persulcatus ticks, and Apodemus speciosus rodents in Japan. Microbiol. Immunol. 38: 413-420.

16. Pisetsky, D. S. 2000. Anti-DNA and autoantibodies. Curr. Opin. Rheumatol. 12: 364-368.

17. Pisetsky, D. S. 2000. The role of bacterial DNA in autoantibody induction. Curr. Top. Microbiol. Immunol. 247: 143-155.

18. Popovsky, M. A., Lindberg, L. E., Syrek, A. L. and Page, P. L. 1988. Prevalence of Babesia antibody in a selected blood donor population. Transfusion 28: 59-61.

19. Saito-Ito, A., Tsuji, M., Wei, Q., He, S., Matsui, T., Kohsaki, M., Arai, S., Kamiyama, T., Hioki, K. and Ishihara, C. 2000. Transfusion-acquired, autochthonous human babesiosis in Japan: isolation of Babesia microti-like parasites with huRBC-SCID mice. J. Clin. Microbiol. 38: 4511-4516.

20. Shiota, T., Kurimoto, H., Haguma, N. and Yoshida, Y. 1984. Studies on Babesia first found in murine in Japan: epidemiology, morphology and experimental infection. Zentralbl. Bakteriol. Mikrobiol. Hyg. Reihe A 256: 347-355.

21. Telford, S. R., III, Gorenflot, A., Brasseur, P. and Spielman, A. 1993. Babesial infections in humans and wildlife. pp. 1-47. In: Parasitic protozoa (Kreier, J. P. ed.), 2nd ed., vol. 5. Academic Press Inc., New York, N. Y.

22. Tsuji, M., Wei, Q., Zamoto, A., Morita, C., Arai, S., Shiota, T., Fugimagari, M., Itagaki, A., Fujita, H. and Ishihara, C. 2001. Human babesiosis in Japan: epizootiologic survey of the rodent reservoir and isolation of new type of Babesia microti-like parasite. J. Clin. Microbiol. 39: 4316-4322.

23. Wei, Q., Tsuji, M., Zamoto, A., Kohsaki, M., Matsui, T., Shiota, T., Teleford, S. R., III and Ishihara, C. 2001. Human 
babesiosis in Japan: isolation of Babesia microti-like parasites from an asymptomatic transfusion donor and from a rodent from an area where babesiosis is endemic. J. Clin. Microbiol. 39: $2178-2183$.

24. White, D. J., Talarico, J., Chang, H. G., Birkhead, G. S., Heimberger, T. and Morse, D. L. 1998. Human babesiosis in New York State: review of 139 hospitalized cases and analysis of prognostic factors. Arch. Intern. Med. 158: 2149-2154.

25. Yokota, S. I., Hirata, D., Minota, S., Higashiyama, T., Kurimoto, M., Yanagi, H., Yura, T. and Kubota, H. 2000. Autoantibodies against chaperonin CCT in human sera with rheumatic autoimmune diseases: comparison with antibodies against other Hsp60 family proteins. Cell Stress Chaperones 5: 337 346. 